\title{
Photoproduction of Neutral Pions off Protons
}

\author{
V. Crede,${ }^{1}$ N. Sparks,${ }^{1}$ A. Wilson, ${ }^{1}$ A.V. Anisovich, ${ }^{2,3}$ J.C.S. Bacelar,${ }^{4}$ R. Bantes,${ }^{5}$ O. Bartholomy, ${ }^{2}$ \\ D. Bayadilov, ${ }^{2,3}$ R. Beck, ${ }^{2}$ Y.A. Beloglazov ${ }^{3}$ R. Castelijns ${ }^{4}$ H. Dutz, ${ }^{5}$ D. Elsner, ${ }^{5}$ R. Ewald, ${ }^{5}$ F. Frommberger,${ }^{5}$ \\ Chr. Funke, ${ }^{2}$ R. Gregor, ${ }^{6}$ A. Gridnev ${ }^{3}$ E. Gutz, ${ }^{2}$ W. Hillert,${ }^{5}$ P. Hoffmeister, ${ }^{2}$ I. Jaegle, ${ }^{7}$ J. Junkersfeld ${ }^{2}$ \\ H. Kalinowsky, ${ }^{2}$ S. Kammer, ${ }^{5}$ Frank Klein, ${ }^{5}$ Friedrich Klein, ${ }^{5}$ E. Klempt,${ }^{2}$ M. Kotulla,,${ }^{7}{ }^{6}$ B. Krusche, ${ }^{7}$ H. Löhner, ${ }^{4}$ \\ I.V. Lopatin ${ }^{3}$ S. Lugert, ${ }^{6}$ D. Menze,${ }^{5}$ T. Mertens, ${ }^{7}$ J.G. Messchendorp,${ }^{4,6}$ V. Metag, ${ }^{6}$ M. Nanova,${ }^{6}$ \\ V.A. Nikonov ${ }^{2}{ }^{3}$ D. Novinski, ${ }^{3}$ R. Novotny, ${ }^{6}$ M. Ostrick,${ }^{5}$ L.M. Pant,${ }^{6}$ H. van Pee, ${ }^{2}$ M. Pfeiffer, ${ }^{6}$ A. Roy,${ }^{6}$ \\ A.V. Sarantsev, ${ }^{2,3}$ S. Schadmand ${ }^{8}$ C. Schmidt,${ }^{2}$ H. Schmieden, ${ }^{5}$ B. Schoch, ${ }^{5}$ S. Shende, ${ }^{4}$ V. Sokhoyan, ${ }^{2}$ \\ A. Süle, ${ }^{5}$ V.V. Sumachev,${ }^{3}$ T. Szczepanek, ${ }^{2}$ U. Thoma,${ }^{2}$ D. Trnka,${ }^{6}$ R. Varma,${ }^{6}$ D. Walther, ${ }^{2,5}$ and Ch. Wendel ${ }^{2}$ \\ (The CBELSA/TAPS Collaboration) \\ ${ }^{1}$ Department of Physics, Florida State University, Tallahassee, Florida 32306, USA \\ ${ }^{2}$ Helmholtz-Institut für Strahlen- und Kernphysik, Universität Bonn, D-53115 Bonn, Germany \\ ${ }^{3}$ Petersburg Nuclear Physics Institute, RU-188350 Gatchina, Russia \\ ${ }^{4}$ KVI, 9747 AA Groningen, The Netherlands \\ ${ }^{5}$ Physikalisches Institut, Universität Bonn, D-53115 Bonn, Germany \\ ${ }^{6}$ II. Physikalisches Institut, Universität Gießen, D-35392 Gießen, Germany \\ ${ }^{7}$ Physikalisches Institut, Universität Basel, CH-4056 Basel, Switzerland \\ ${ }^{8}$ Institut für Kernphysik, Forschungszentrum Jülich, D-52428 Jülich, Germany
}

(Dated: Received: November 10, 2011/ Revised version:)

\begin{abstract}
Photoproduction of neutral pions has been studied with the CBELSA/TAPS detector in the reaction $\gamma p \rightarrow p \pi^{0}$ for photon energies between 0.85 and $2.50 \mathrm{GeV}$. The $\pi^{0}$ mesons are observed in their dominant neutral decay mode: $\pi^{0} \rightarrow \gamma \gamma$. For the first time, the differential cross sections cover the very forward region, $\theta_{\text {c.m. }}<60^{\circ}$. A partial-wave analysis of these data within the Bonn-Gatchina framework observes the high-mass resonances $G_{17}(2190), D_{13}(2080)$, and $D_{15}(2070)$.
\end{abstract}

PACS numbers: 13.60.Le Meson production, 13.60.-r Photon and charged-lepton interactions with hadrons, 13.75.Gx Pion-baryon interactions, 14.40.Aq $\pi, K$, and $\eta$ mesons, 25.20.Lj Photoproduction reactions

\section{INTRODUCTION}

Spectroscopy has long provided essential information for scientists trying to understand the nature of particles. The spectroscopy of the hydrogen atom revealed the quantum nature of the interactions between particles at small distance scales and led to the development of quantum electrodynamics (QED). Exploration of the excited states of hadrons opens a door into the nature of nonperturbative quantum chromodynamics (QCD). By mapping out the excitation spectra of hadrons, information is gained about their effective degrees of freedom and the effective forces that lead to confinement. A both particularly challenging and interesting task is to understand the spectra of baryons. Although symmetric quark models, describing the baryon with three constituent quark degrees of freedom, are good at predicting many properties for many of the low-lying baryons (especially the ground states), there is a large disagreement between the number of excited states predicted by these models and the number that has been observed at and, in particular, above masses of about $1.8 \mathrm{GeV} / c^{2}$. Attempts at finding a physical mechanism that can account for this discrepancy have not thus far yielded a satisfactory answer, and the higher mass regions of the excitation spectra remain largely unexplored: Most known baryon resonances have masses smaller than $2 \mathrm{GeV} / c^{2}$ and were discovered in elastic $\pi N$ scattering experiments. Many of the missing or hitherto unobserved baryon resonances are predicted to couple only weakly to the $\pi N$ channel but exhibit large couplings to $\gamma N$. In recent years, many different laboratories worldwide (Jefferson Laboratory, ELSA, MAMI, GRAAL, SPring-8, etc.) have measured differential cross sections and polarization observables for a large variety of final states in electromagnetically induced reactions. A good review is given in 1$]$.

Although many of the missing baryon resonances may have a small coupling to $\pi N$, it is still important to study pion photoproduction. Doing so may confirm or reject resonances seen in elastic pion-nucleon scattering, and it could still provide evidence for unexplored resonances. New resonances found in reactions such as $\gamma N \rightarrow \pi N$ are expected to have masses larger than about $1.8 \mathrm{GeV} / c^{2}$, although the higher-mass resonance contributions are expected to be more important in double-meson photoproduction, by sequentially decaying via emission of a $\pi$ or $\eta$ meson and populating intermediate states.

Excited baryons are broad and overlapping, making it difficult to extract their properties by studying isolated reaction channels and cross sections alone. Polarization observables can provide access to small resonance contributions to a final state, so that missing resonances may still be found by studying these in pion photoproduction. However, both unpolarized cross sections and polarization observables are needed to maximally constrain and uniquely determine the scattering amplitude for a given 


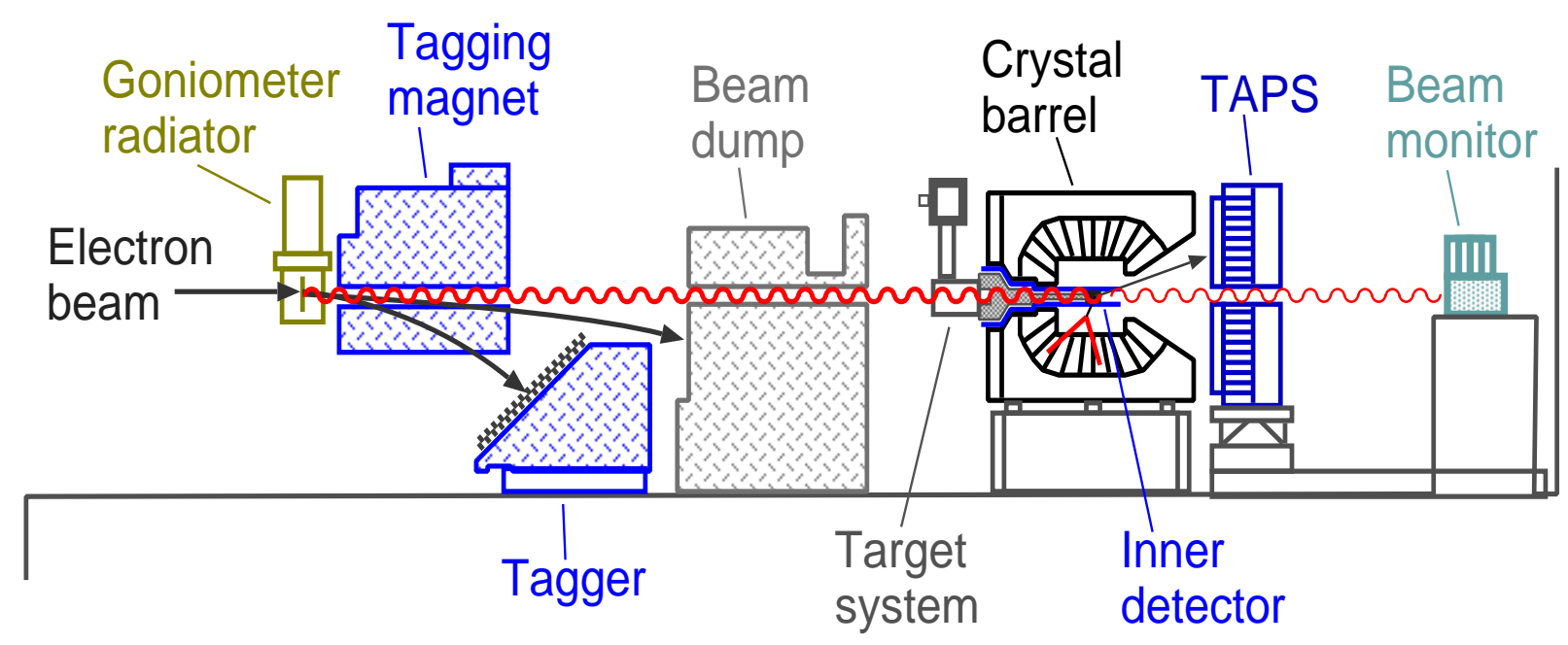

FIG. 1: (Color online) Cross section of the CBELSA/TAPS setup (not to scale). The electron beam delivered by the accelerator ELSA enters from the left side and hits the copper radiator (for the data presented here) in front of the tagger magnet.

process. Additional physical constraints are provided by performing an analysis of these observables for multiple reaction channels simultaneously, and to succeed in its description of the contributing resonances, the combined analysis of these data must accurately describe the nonresonant behavior of the observables. Previous experiments have shown that $t$-channel contributions are important in pion photoproduction at high energies, where the missing resonances are expected. Thus further study of the pion photoproduction cross section, particularly at forward angles, is very interesting.

Recent measurements of differential cross sections in pion photoproduction have made significant contributions to the world database, but these measurements have limited coverage at very forward center-of-mass polar angles of the pion, $\theta_{\text {c.m. }}$. The recent CB-ELSA [2, 3] and CLAS data [4] on $\gamma p \rightarrow p \pi^{0}$ cover angles down to approximately $30^{\circ}$ and $40^{\circ}$ in $\theta_{\text {c.m. }}$., respectively. They show discrepancies, where the respective differential cross sections are observed to increase at different rates as one moves to more forward angles for the incoming photon energy range 1300 to $2000 \mathrm{MeV}$. Our new CBELSA/TAPS data, presented here, extend the coverage of the forward region and will help understand the behavior of the $\pi^{0}$ photoproduction cross section in this kinematic region.

In this paper, we present differential cross sections for the reaction:

$$
\gamma p \rightarrow p \pi^{0}, \quad \text { where } \pi^{0} \rightarrow 2 \gamma
$$

We studied, in particular, the forward region; a similar study was published for the beam asymmetries $\Sigma$ in the same reaction [5].

The paper has the following structure. Section 1 summarizes recent results on $\pi^{0}$ photoproduction. A brief introduction to the CBELSA/TAPS experimental setup is given in Section III. The data reconstruction and event selection are discussed in Section IV] and the extraction of cross sections is described in Section $\mathrm{V}$ Experimental results are finally presented in Section $\mathrm{VI}$, and results of a partial-wave analysis are discussed in Section VII

\section{PREVIOUS RESULTS}

Cross section data for $\pi^{0}$ photoproduction were obtained and studied at many different laboratories over a wide kinematic range 2 2 $4,6,18]$. A review of the main data sets published before 2005 and a corresponding comparison of their coverage in energy and solid angle can be found in [3]. The new data presented in this paper extend previous data by covering the very forward polar angles with an electromagnetic calorimeter, TAPS.

In 2005, the CB-ELSA collaboration at Bonn, Germany, presented high-statistics results on the photoproduction of $\pi^{0}$ mesons using the Crystal Barrel detector for incident photon energies from 300 to $3000 \mathrm{MeV}$ [2]. Above energies of $1300 \mathrm{MeV}$, the data cover an angular range from about $30^{\circ}$ to $140^{\circ}$, which corresponds to about $-0.75<\cos \theta_{\text {c.m. }}<0.85$.

The GRenoble Anneau Accelerateur Laser (GRAAL) collaboration at ESRF in Grenoble, France, measured differential cross sections over a wide angular range from 550 to $1500 \mathrm{MeV}$ [15], though still limited to $\cos \theta_{\text {c.m. }}<0.7$ or approximately $\theta_{\text {c.m. }}>45^{\circ}$. At the GRAAL facility, Compton backscattering of lowenergy photons off ultrarelativistic electrons reached almost $100 \%$ beam polarization at the Compton edge.

More recently, the LEPS collaboration at SPring-8 in Hyogo, Japan, published results for higher photon energies between $E_{\gamma}=1500$ and $2400 \mathrm{MeV}$ [16]. For the first time, the data cover the $\pi^{0}$ backward angles between $-1<\cos \theta_{\text {c.m. }}<-0.6$ or about $125^{\circ}<\theta_{\text {c.m. }}<180^{\circ}$. Backward-Compton scattering was applied using Ar-ion laser photons with a 351-nm wavelength. 

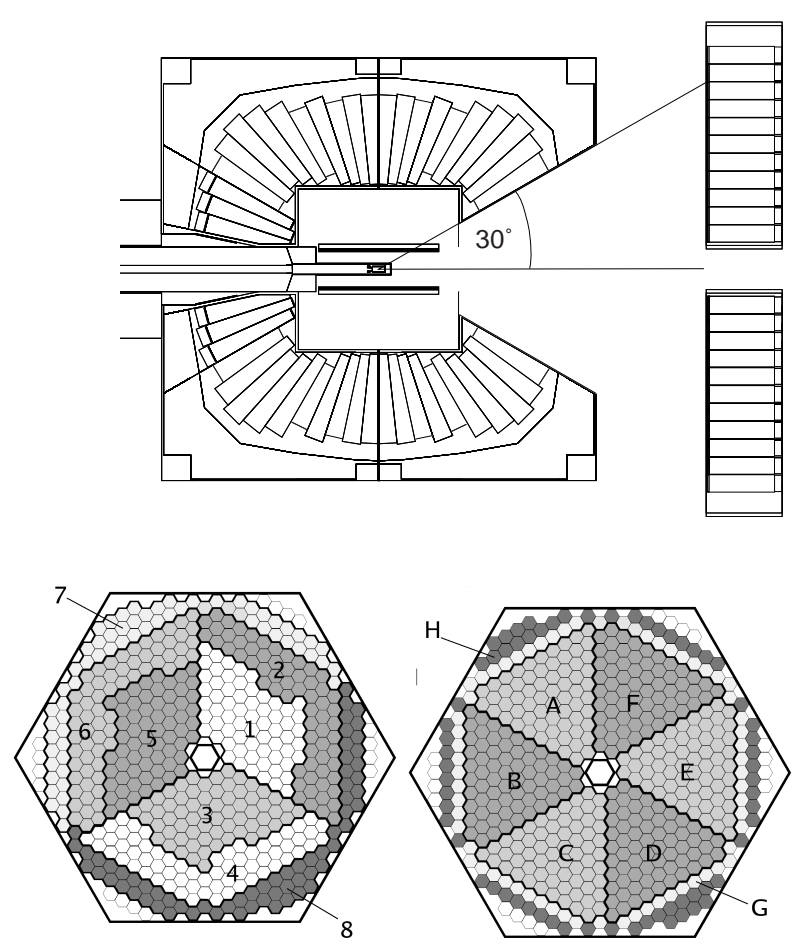

FIG. 2: (Top) Schematic drawing of the liquid-hydrogen target, scintillating-fiber detector, Crystal Barrel and TAPS calorimeters. (Bottom) Front view of TAPS: The left side shows the logical segmentation for the LED-low trigger; the right side shows the logical segmentation for the LED-high trigger (see text for more details).

At Jefferson Lab, Newport News, VA, differential cross sections for the reaction were measured using the CEBAF Large Acceptance Spectrometer (CLAS) and a tagged photon beam with energies from 0.675 to $2.875 \mathrm{GeV}$ [4]. The cross-section data cover an angular range from $\theta_{\text {c.m. }} \approx 40^{\circ}$ to $150^{\circ}$, corresponding to approximately $-0.85<\cos \theta_{\text {c.m. }}<0.75$.

\section{EXPERIMENTAL SETUP}

The experiment was conducted using the Crystal Barrel [19] and TAPS [20, 21] electromagnetic calorimeters at the electron stretcher accelerator ELSA [22], which is located at the University of Bonn in Germany. Figure 1 contains a schematic of the experimental setup.

A 3.175-GeV electron beam was extracted (by slow resonant extraction) from ELSA. The electrons were passed through a thin copper radiator, which had a thickness of (3/1000) $X_{R}$ (radiation length), where a fraction of them underwent bremsstrahlung. These electrons were deflected by a dipole magnet into the tagger detector system (tagger), which consisted of 480 scintillating fibers above 14 scintillation counters in a partly overlapping configuration. Good knowledge of the magnetic field and the position of the hit in the tagger allowed the momentum and energy of each electron to be determined. The tagged-photon energy is given by the difference between the incoming electron energy and the electron energy after bremsstrahlung. Electrons that did not undergo bremsstrahlung were deflected at small angles into a beam dump located behind the tagger. Tagged photons had energies ranging from 0.5 to $2.9 \mathrm{GeV}$; the higher energy part of this bremsstrahlung spectrum had an energy resolution of about $2 \mathrm{MeV}$, while the lower part had an energy resolution of approximately $25 \mathrm{MeV}$.

The energy calibration of the photon tagging system was determined in simulations using the known positions of the scintillating fibers and the measured magnetic field map, and approximated by a polynomial. Measurements with a known (lower-energy) ELSA beam allowed for a cross-check of the calibration; this involved guiding a lowcurrent beam directly into the tagger, while holding the magnetic field constant. Corrections to the original polynomial were determined from this procedure.

Tagged photons continued along the beam line toward a liquid-hydrogen target, which was $5 \mathrm{~cm}$ in length and $3 \mathrm{~cm}$ in diameter, located at the center of the Crystal Barrel (CB) calorimeter (see Fig. 1). Some of these photons interacted with protons within the target to produce a proton and multiple photons in the final state, the photons resulting from the decay of neutral mesons. Final-state photons were detected by the two electromagnetic calorimeters (CB and TAPS), which together almost covered the full $4 \pi$ solid angle ( $>98 \%$ coverage). The CB detector in its CBELSA/TAPS configuration of 2002/2003 consisted of $1290 \mathrm{CsI}(\mathrm{Tl})$ crystals, which had a trapezoidal shape and were oriented toward the target, with a thickness of $16 X_{R}$, and covered the polar angles from $30^{\circ}$ to $168^{\circ}$, while TAPS consisted of 528 hexagonal $\mathrm{BaF}_{2}$ crystals with a length of approximately $12 X_{R}$, and covered the polar angles from $5^{\circ}$ to $30^{\circ}$; both covered the full azimuthal circle. TAPS was configured as a hexagonal wall and served as the forward endcap of the CB. The $\mathrm{CB}$ originally covered polar angles down to $12^{\circ}$ and was made of 26 rings [19]. Downstream rings $11-13$ of the CB were removed to allow for a tight fit with TAPS (see top of Fig. 2). Forward-going protons were detected by plastic scintillators ( $5 \mathrm{~mm}$ thick) located in front of each TAPS module; the other protons were detected by a three-layer scintillating fiber detector, which closely surrounded the target [23].

Photons that did not interact with the target material continued further along the beam line through a hole in TAPS and finally arrived at the beam monitor, a total absorption Cerenkov counter, which consisted of an array of nine lead glass crystals. The number of photons counted here was vital to the determination of the photon flux.

The first-level trigger was provided by the fast response of the TAPS modules. A cellular logic called FAstCluster Encoder (FACE), which counted the number of clusters in the barrel, formed the second-level trigger. 

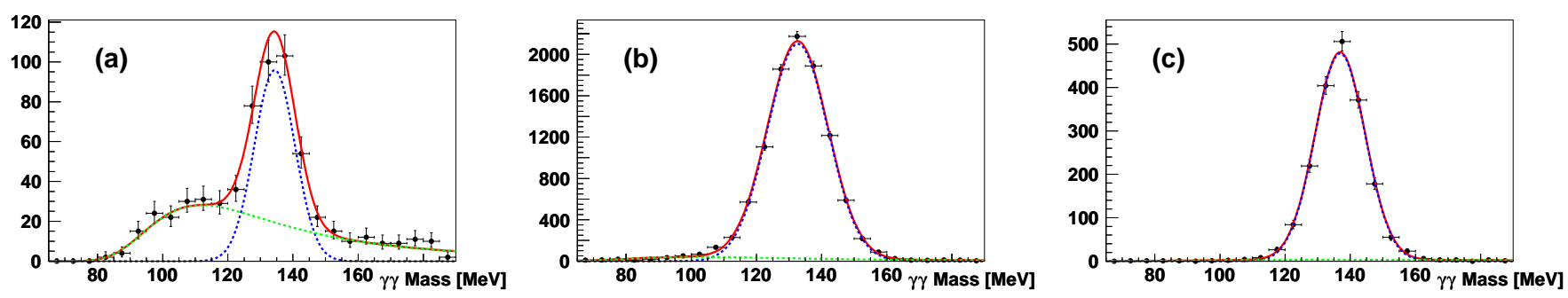

FIG. 3: (Color online) Invariant $\gamma \gamma$ mass spectra for the reaction $\gamma p \rightarrow p \gamma \gamma$ for $E_{\gamma} \in[875,900] \mathrm{MeV}$ and for $(\mathrm{a}) \theta_{\text {c.m. }} \in\left[0^{\circ}, 10^{\circ}\right]$, (b) $\theta_{\text {c.m. }} \in\left[40^{\circ}, 50^{\circ}\right]$, and (c) $\theta_{\text {c.m. }} \in\left[160^{\circ}, 170^{\circ}\right]$. CL cuts were applied at $10^{-2}$. The red solid line denotes the signal Gaussian, the green dotted line the Landau distribution for the background description, and the blue dotted line shows the background subtracted distribution. The background is essentially negligible for $\theta_{\text {c.m. }}>50^{\circ}$.

The trigger required at least one hit in TAPS above a high-energy threshold (LED-high) and at least one FACE cluster, or it required at least two hits in TAPS in different segments above a low-energy threshold (LED-low). The logical LED-low and LED-high segmentation of the trigger is shown in Fig. 2. Topologically, reaction (1) events satisfying the first condition have either a fast proton in TAPS (and a backward-going pion in the CB) or one high-energy photon in TAPS and one photon in the CB (from a forward-going pion), while those that satisfy the second condition have a pion in TAPS, together leaving a hole in the pion acceptance at intermediate angles.

\section{PREPARATION OF FINAL STATE}

The data presented here were recorded from October 2002 until November 2002 in two run periods at ELSA beam energies of $3.175 \mathrm{GeV}$. For this analysis, only the photon energy range up to $2.55 \mathrm{GeV}$ was used since the tagger consisted of scintillating fibers covering energies up to this energy and providing timing information, while simple hit/miss wires covered photon energies above $2.55 \mathrm{GeV}$. These data were also used for previously published analyses on a variety of different final states 24 26].

This section discusses the event reconstruction and selection of the $\pi^{0}$ photoproduction channel: reaction (11). A total number of $2.21 \times 10^{6} \pi^{0}$ events was observed, covering invariant masses from 1570 to $2360 \mathrm{MeV} / c^{2}$.

\section{A. Event Reconstruction}

The event selection was carried out in close analogy to the study of the $\eta$ photoproduction channel. Further details on the event reconstruction and the use of kinematic fitting in this analysis can be found in [26].

Events were analyzed for which only the two final-state photons (from the decay of the pion, $\pi^{0} \rightarrow \gamma \gamma$ ) or all final-state particles $(\gamma \gamma+p)$ were detected. The protons were identified by using the photon-veto $\mathrm{BaF}_{2}$ detectors of TAPS or the inner scintillating fiber detector inside the
CB. A CB cluster is assigned to a charged particle if the trajectory from the target center to the barrel hit forms an angle of less than $20^{\circ}$ with a trajectory from the target center to a hit in the inner detector. The resolution for proton clusters in the calorimeters is worse than that for photon clusters because, on average, proton clusters are much smaller than photon clusters. Proton identification was only used to separate protons from photons, but the proton four-vector was determined from the event kinematics through missing-proton kinematic fitting.

Time accidental background was reduced by demanding a prompt coincidence between an electron in the tagger and a particle in TAPS; if this particle was a photon, it required a time difference within $\pm 3 \mathrm{~ns}$, while for a proton a time difference within -5 to +15 ns was required. Two to three tagger photons, at most, survived the initial timing cut. A missing-proton kinematic fit was used to separate the best initial-state photon from the remaining candidates in the event. This $1 \mathrm{C}$ kinematic fit to the hypothesis $\gamma p \rightarrow p_{\text {miss }} \gamma \gamma$, which only required fourmomentum conservation, was performed for each initial photon candidate of an event passing the initial timing cut, and the candidate with the largest confidence level (CL) or $\chi^{2}$ probability was selected. Finally, only events with a CL $>1 \%$ were accepted for further analysis. No mass constraints were used in the kinematic fitting.

In the final step of the event reconstruction, we removed events that are not $p\left(\pi^{0} \rightarrow \gamma \gamma\right)$ final states (background) from the event sample. This was done by interpolating the shape of the background in the signal region based on the distribution of events outside the signal region. The data were binned, and maximum likelihood fits of a signal plus background hypothesis to the data were performed. A Gaussian line shape was used for the signal, and a Landau distribution was used for the background. Typical $\gamma \gamma$ mass distributions are shown in Fig. 3 for $E_{\gamma} \in[875,900] \mathrm{MeV}$. The size of the background at very forward angles is substantial [Fig. 3.(a)]; it is much smaller, on average, in the backward bins at less than 4\% [Fig. 3(c)]. Mass resolutions (standard deviations of Gaussian signals) extracted from the data agree within $1 \mathrm{MeV} / \mathrm{c}^{2}$ with those from Monte Carlo simulations. 


\section{B. Monte Carlo Simulations}

The performance of the detector was simulated in GEANT3-based Monte Carlo studies. We used a program package that has been built upon a program developed for the CB-ELSA experiment. The Monte Carlo program accurately reproduces the response of the TAPS and CB crystals to photons.

The acceptance for reaction (11) was determined by simulating events that were evenly distributed over the available phase space. The tagging and timing of initial-state photons were not simulated. The Monte Carlo events were analyzed by using the same reconstruction criteria as were also applied to the (real) measured data. The same 1C-hypothesis was tested in the kinematic fits, and events were selected with the same CL cuts. The acceptance is defined as the ratio of the number of reconstructed to generated Monte Carlo events,

$$
A_{\gamma p \rightarrow p X}=\frac{N_{\mathrm{rec}, \mathrm{MC}}}{N_{\text {gen }, \mathrm{MC}}} \quad\left(X=\pi^{0}\right) .
$$

In the analysis presented here, we have applied an acceptance cut of at least $5 \%$ on $\left(E_{\gamma}, \cos \theta_{\text {c.m. }}\right)$ bins, as well as an acceptance cut of at least $1 \%$ on $\left(E_{\gamma}, \theta_{\text {c.m. }}\right)$ bins, and removed the other data points from the analysis. Different values of the acceptance cut were used for the two binnings to provide comparable statistics over a large angular range.

\section{DETERMINATION OF CROSS SECTIONS}

The differential cross sections for this analysis have been determined according to

$$
\frac{\mathrm{d} \sigma}{\mathrm{d} \Omega}=\frac{N_{\pi^{0} \rightarrow \gamma \gamma}}{A_{\pi^{0} \rightarrow \gamma \gamma}} \frac{1}{N_{\gamma} \rho_{\mathrm{t}}} \frac{1}{\Delta \Omega} \frac{1}{\frac{\Gamma_{\pi^{0} \rightarrow \gamma \gamma}}{\Gamma_{\text {total }}}},
$$

where

$$
\begin{aligned}
& \rho_{\mathrm{t}}: \text { target area density } \\
& \mathrm{N}_{\pi^{0} \rightarrow \gamma \gamma}: \text { number of reconstructed data events } \\
& \text { in an }\left(E_{\gamma}, \cos \theta_{\text {c.m. }}\right) \text { or }\left(E_{\gamma}, \theta_{\text {c.m. }}\right) \text { bin } \\
& N_{\gamma}: \text { number of beam photons in an }\left(E_{\gamma}\right) \text { bin } \\
& \mathrm{A}_{\pi^{0} \rightarrow \gamma \gamma}: \text { acceptance in an }\left(E_{\gamma}, \cos \theta_{\text {c.m. }}\right) \text { or }\left(E_{\gamma}, \theta_{\text {c.m. }}\right) \text { bin } \\
& \Delta \Omega: \text { solid-angle interval } \Delta \Omega=2 \pi \Delta \cos \left(\theta_{\text {c.m. }}\right) \\
& \frac{\Gamma_{\pi^{0} \rightarrow \gamma \gamma}}{\Gamma_{\text {total }}}: \text { decay branching fraction. }
\end{aligned}
$$

The target area density, that is, the number of atoms in the target material per cross-sectional area (orthogonal to the photon beam), is given by

$$
\rho_{\mathrm{t}}=2 \frac{\rho\left(\mathrm{H}_{2}\right) N_{A} L}{M_{\mathrm{mol}}\left(\mathrm{H}_{2}\right)}=2.231 \cdot 10^{-7} \mu \mathrm{b}^{-1},
$$

where $\rho\left(\mathrm{H}_{2}\right)=0.0708 \mathrm{~g} / \mathrm{cm}^{3}$ is the density, $M_{\mathrm{mol}}=$ $2.01588 \mathrm{~g} / \mathrm{mol}$ is the molar mass of liquid $\mathrm{H}_{2}, N_{A}=$
$6.022 \cdot 10^{23} \mathrm{~mol}^{-1}$ is the Avogadro number, and $L=5.275$ $\mathrm{cm}$ is the length of the target cell. The factor of two accounts for the molecular composition of hydrogen $\left(\mathrm{H}_{2}\right)$.

The number of events in an $\left(E_{\gamma}, \cos \theta_{\text {c.m. }}\right)$ or $\left(E_{\gamma}\right.$, $\left.\theta_{\text {c.m. }}\right)$ bin comprises events with two or three final-state particles (at least $2 \gamma^{\prime}$ 's). The proton can be "missing", but events with and without a detected proton are treated in the same way in the event reconstruction. For $\cos \theta_{\text {c.m. }}<0.0$, the event kinematics requires that the proton is used in the (TAPS) trigger. Thus, the detection efficiency of forward-going protons needs to be reasonably well understood. This has been shown earlier for CBELSA/TAPS results on $\eta$ photoproduction; more details on the proton trigger can be found in [26].

The interval of the solid angle is given by $\Delta \Omega=$ $2 \pi \Delta \cos \left(\theta_{\text {c.m. }}\right)$, with $\Delta \cos \left(\theta_{\text {c.m. }}\right)$ describing the width of the angular bins. It was chosen to be 0.1 for the $\pi^{0}$ data presented here. For the representation of the differential cross sections in terms of the angle $\theta_{\text {c.m. }}$. directly, a bin width of $10^{\circ}$ was chosen. Energy bins were defined by considering statistics and ensuring a good comparability with other experiments. A total of 42 bins is presented in energy steps of $25 \mathrm{MeV}$ for $E_{\gamma} \in[850,1300] \mathrm{MeV}$ and $50 \mathrm{MeV}$ for $E_{\gamma} \in[1300,2500] \mathrm{MeV}$.

The number of observed $\pi^{0}$ mesons needs to be corrected for unseen decay modes. The partial-decay branching fraction used to correct the measured cross sections is taken from [27]: $\operatorname{BR}\left(\pi^{0} \rightarrow \gamma \gamma\right)=0.98823 \pm$ 0.00034 .

\section{A. Normalization}

The CBELSA/TAPS tagging hodoscope consisted of 480 scintillating fibers above 14 partially overlapping scintillation counters (tagger bars). The photon flux was measured directly in the experiment and determined according to

$$
N_{\gamma}=\alpha \cdot P_{\gamma} \cdot N_{\mathrm{scaler}}^{\mathrm{fiber}}
$$

where the parameter $\alpha$ accounts for the (fiber)-cluster reconstruction in the tagger, which has to be performed in the same way as for real hadronic events. $P_{\gamma}$, the photon definition probability, denotes the probability that a real photon is emitted along the beam axis in the tagger and traverses the liquid hydrogen target; it is determined from Tagger-Or-Runs, separate data runs utilizing a minimum-bias trigger. $N_{\text {scaler }}^{\text {fiber }}$ is the number of free hardware counts for the individual fibers corrected for the lifetime of the detector. Scalers are recorded in scaler events, which were accumulated with a minimumbias trigger at a rate of $1 \mathrm{~Hz}$ during regular data taking. This trigger required only a hit in the tagger and was thus independent of hadronic cross sections.

The $P_{\gamma}$ error is assumed to dominate the total error of the photon flux, which has a strong dependence on the efficiency of the beam monitor at the end of the beam line (Fig. 1). By varying the background subtraction 
of noncoincident tagger-beam monitor hits, $P_{\gamma}$ was determined to be $0.639 \pm 0.002_{\text {stat. }} \pm 0.05_{\text {sys. }}$ 26]. This value agrees with determinations from multiple TaggerOr-Runs at different incoming photon rates. An overall error of $10 \%$ has been assigned to the photon flux determination and has been included in Figs. 4 and 5 .

\section{B. Systematic Uncertainties}

The statistical errors are determined from the number of events in each $\left(E_{\gamma}, \cos \theta_{\text {c.m. }}\right)$ or $\left(E_{\gamma}, \theta_{\text {c.m. }}\right)$ bin. Statistical errors are shown for all data points; systematic uncertainties are given as error bands at the bottom of each plot.

The systematic errors include uncertainties from the position of the liquid hydrogen target and a possible offset of the photon beam. The position of the target cell was determined from kinematic fitting by comparing the off-zero displacement of different pull distributions to Monte Carlo simulations. It was found to be shifted upstream by $0.65 \mathrm{~cm} \mathrm{[3]}$. The corresponding systematic errors were determined by varying the target position in the Monte Carlo $( \pm 1.5 \mathrm{~mm})$ and evaluating changes in the reextracted differential cross sections. The errors show an angular dependence, but are $3-4 \%$ on average and $<7 \%$ at most in the very forward region, $\theta_{\text {c.m. }}<50^{\circ}$. The photon beam was assumed to be shifted by less than $2 \mathrm{~mm}$ off axis at the target position. The errors of the decay branching fractions are negligible.

The reconstruction of photons from the decay of neutral mesons and the identification of final states requires a sequence of cuts including the use of kinematic fitting. In [26], it was discussed in detail that the reconstruction of $\eta$ mesons from final states with two and six photons leads to compatible results for the $\eta$ differential cross sections. The reconstruction of $\pi^{0}(\rightarrow \gamma \gamma)$ mesons is very similar to the reconstruction of $\eta$ mesons in the same two-photon decay mode and for the $\pi^{0}$ analysis discussed here, the same data sets, software packages, and calibration were used. This shows the good understanding of the detector response to multi-photon final states. An overall $\pm 5.7 \%$ error is assigned to the reconstruction efficiency as determined in [28]. An additional 3\% systematic error accounts for the slightly different effects of CL cuts on data and Monte Carlo events. The uncertainty of the proton trigger has been estimated from the small disagreement of the differential $\eta$ cross sections using the $\eta \rightarrow 2 \gamma$ and $\eta \rightarrow 3 \pi^{0} \rightarrow 6 \gamma$ decay channels for $E_{\gamma}<1 \mathrm{GeV}$ and $\cos \theta_{\text {c.m. }}<0.0$ where the proton trigger dominates over triggers caused by photons.

All the discussed contributions to the systematic error have been added quadratically to give the total systematic uncertainty for this reaction.

\section{EXPERIMENTAL RESULTS}

Figure 4 shows the $\gamma p \rightarrow p \pi^{0}$ differential cross sections plotted versus $\cos \theta_{\text {c.m. }}$. of the $\pi^{0}$, using $\cos \theta_{\text {c.m. }}$. bins of width 0.1 . To facilitate the comparison with our previous CB-ELSA data, we have chosen energy bins of $25 \mathrm{MeV}$ for the energy range $E_{\gamma} \in[850,1300] \mathrm{MeV}$ and energy bins of $50 \mathrm{MeV}$ for the energy range $E_{\gamma} \in[1300,2500] \mathrm{MeV}$. The data cover the very forward region. We have excluded those data points in this analysis that show a Monte Carlo acceptance of $<5 \%$. The error bars are statistical only; the total systematic error is given as error bands at the bottom of each plot. The angular distributions exhibit a region of zero acceptance around approximately $-0.3<\cos \theta_{\text {c.m. }}<0.3$, depending on energy, because of the trigger conditions during the data taking. We observed a small drop of the cross section for $E_{\gamma} \in[1075,1100] \mathrm{MeV}$, owing to an increased photon flux determined for one of the tagger fibers defining this energy bin; the reason for this increase is unknown, and thus these data are not shown. Resonance production is clearly observed up to high energies, as can be seen from variations in the differential cross sections over the full angular range up to high energies. Above $E_{\gamma}>1.5 \mathrm{GeV}$, the development of a forward peak indicates important contributions from $t$-channel exchange, which becomes dominant above incoming energies of $2.2 \mathrm{GeV}$.

Figure 5 shows the same $\gamma p \rightarrow p \pi^{0}$ differential cross sections plotted versus $\theta_{\text {c.m. }}$. of the $\pi^{0}$ to illustrate the improvement of the data coverage in the forward direction. The width of each $\theta_{\text {c.m. }}$. bin is $10^{\circ}$. This change of the data representation and the modified binning required a new analysis and subtraction of the background under the $\pi^{0}$ peaks in the mass spectra as well as a new investigation of the systematic errors. Again, the total systematic error is given as error bands at the bottom of each plot. For this representation of the data, we have excluded data points that show a Monte Carlo acceptance of $<1 \%$. Figure 6 shows the differential cross sections at three different incoming photon energies to facilitate the comparison with a variety of other data sets.

\section{A. Comparison with CB-ELSA and CLAS Data}

A more detailed comparison of the results in the form of cross section ratios of this analysis to other data sets is shown in Fig. 7. The red open circles represent the forward direction, $\cos \theta_{\pi^{0}}>0$, and the solid blue squares the backward direction, $\cos \theta_{\pi^{0}}<0$. In Fig. 7(a), we compare the new CBELSA/TAPS data of this analysis with the previous CLAS data [4] and in Fig. 7(b), with the previous CB-ELSA data 2]. In Fig. 7(c), a comparison between CB-ELSA and CLAS is shown. Since the earlier CB-ELSA data switch from a $50-\mathrm{MeV}$ to a $100-$ $\mathrm{MeV}$ energy binning at high energies owing to statistical limitations, the distributions in panels (b) and (c) are cut off at an incoming photon energy of $2.3 \mathrm{GeV}$. 


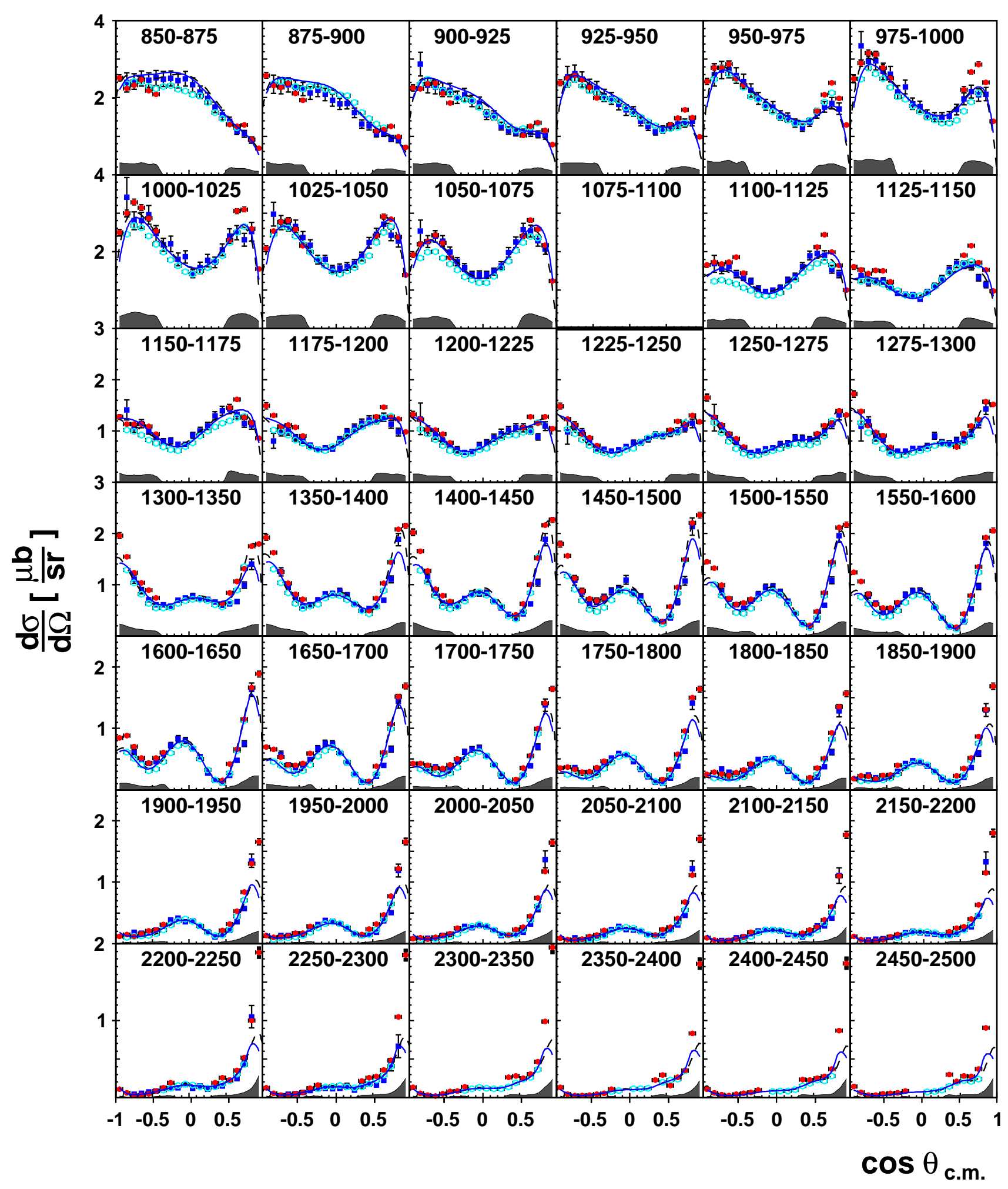

FIG. 4: (Color online) Differential cross sections for $\gamma p \rightarrow p \pi^{0}$ from CBELSA/TAPS $(\bullet)$ plotted versus cos $\theta_{\text {c.m. }}$ For comparison, CB-ELSA data 2] are represented by (-) and CLAS data [4] by ( 0 ). Note that the CLAS cross sections were extracted for $50-\mathrm{MeV}$-wide energy bins; for $E_{\gamma}<1.3 \mathrm{GeV}$, the same CLAS results are thus shown in the corresponding 25-MeV-wide bins chosen for this analysis. The solid blue line shows the solution of the Bonn-Gatchina partial-wave analysis [29], and the dashed black line represents the current SAID solution (SP09) [30]. The data points include statistical errors only; the total systematic error is given as error bands at the bottom of each plot. 


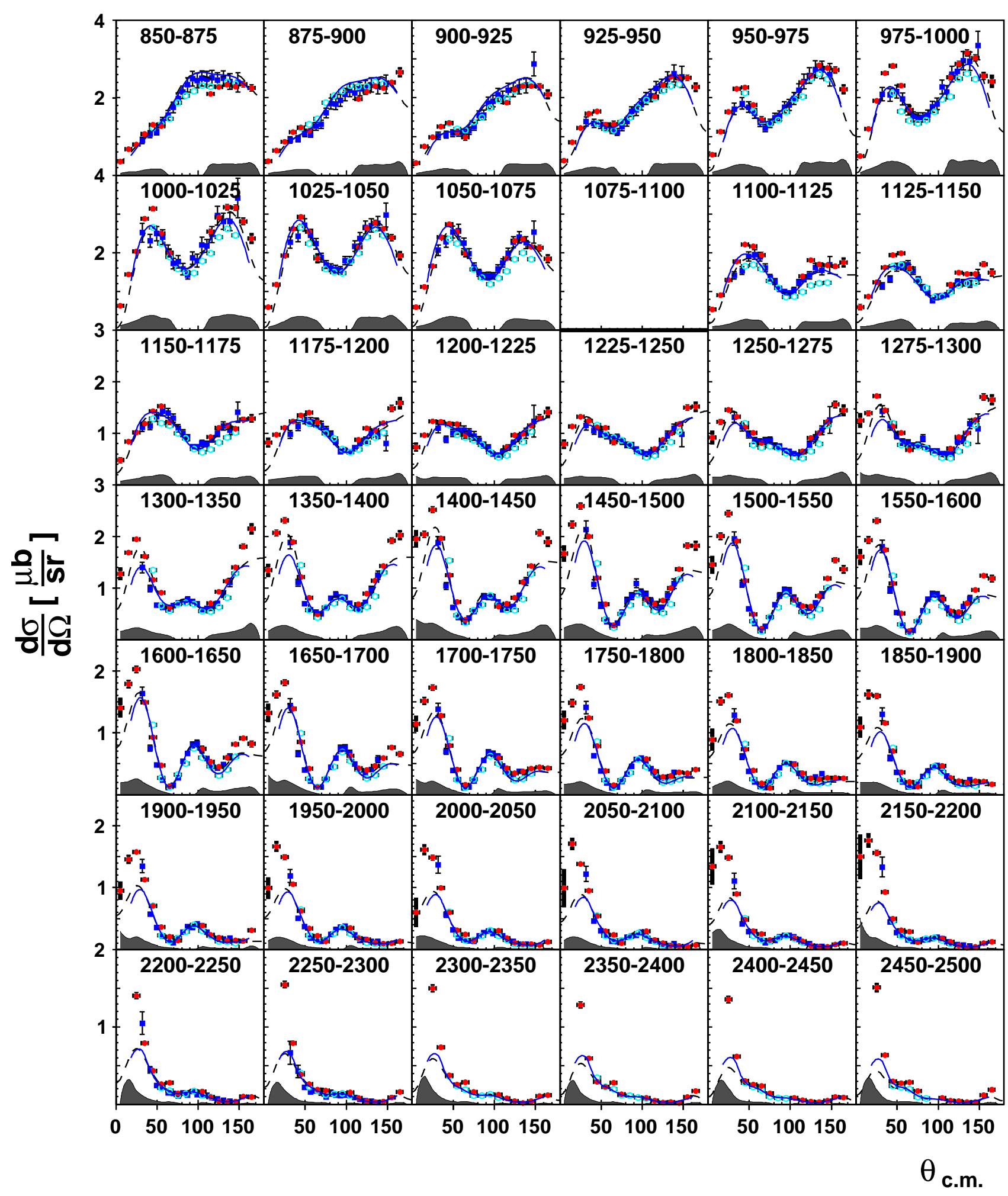

FIG. 5: (Color online) Differential cross sections for $\gamma p \rightarrow p \pi^{0}$ from CBELSA/TAPS $(\bullet)$ plotted versus $\theta_{\text {c.m. }}$. For comparison, CB-ELSA data 2] are represented by (-) and CLAS data [4] by (०). Note that the CLAS cross sections were extracted for $50-\mathrm{MeV}$-wide energy bins; for $E_{\gamma}<1.3 \mathrm{GeV}$, the same CLAS results are thus shown in the corresponding 25-MeV-wide bins chosen for this analysis. The solid blue line shows the solution of the Bonn-Gatchina partial-wave analysis [29], and the dashed black line represents the current SAID solution (SP09) 30]. The data points include statistical errors only; the total systematic error is given as error bands at the bottom of each plot. 


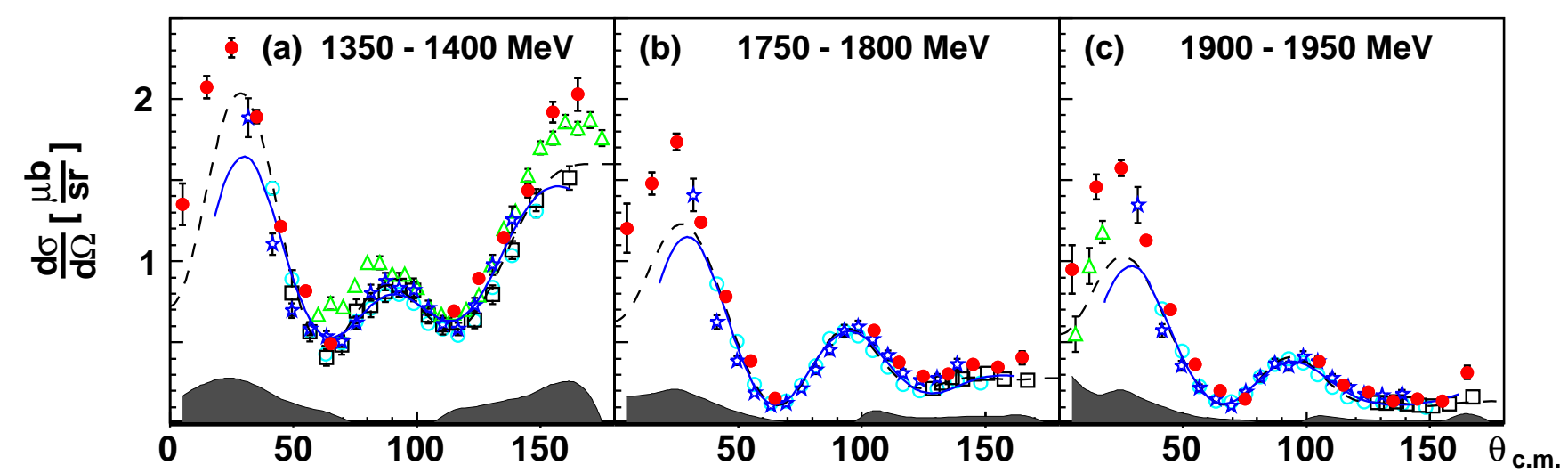

FIG. 6: (Color online) The differential cross sections for $\gamma p \rightarrow p \pi^{0}$ at $E_{\gamma} \in[1350,1400] \mathrm{MeV}$ (left), $E_{\gamma} \in[1750,1800] \mathrm{MeV}$ (center), and $E_{\gamma} \in[1900,1950] \mathrm{MeV}$ (right). The angle shown is the pion center-of-mass scattering angle. The experimental data are from the CBELSA/TAPS measurements presented here (filled circles •), CB-ELSA 2] (open stars «), GRAAL (left) [15] (open boxes $\square$ ), SPring-8 (center and right) [16] (open boxes $\square$ ), CLAS [4] (open circles $\bigcirc$ ), Althoff et al. (left) [17] (open triangles $\triangle$ ), and Brefeld et al. (right) [18] (open triangles $\triangle$ ). The dashed lines represent the current SAID solution (SP09) [30], and the solid lines represent the latest solution of the Bonn-Gatchina PWA 29], which includes the new data presented here. The total systematic error is given again as an error band at the bottom of each figure.

Figure 8 shows the excitation functions for the reaction $\gamma p \rightarrow p \pi^{0}$ at fixed values of the pion center-ofmass scattering angle using a logarithmic scale. We also added available data points in the backward direction from LEPS [16]. The agreement with LEPS is good and confirms the weak energy dependence of the cross section found above $2 \mathrm{GeV}$ and at very backward angles. A very similar, mostly flat distribution is observed at very forward angles.

In a direct comparison, our new data show an overall good agreement with the older CB-ELSA data within systematic errors [Fig. (7)(b)]. While our new data show a steeper slope of the forward ( $t$-channel) rise than the earlier CB-ELSA results, the most forward CB-ELSA point at $\theta_{\text {c.m. }} \approx 35^{\circ}$ is almost perfectly matched for all energies, indicating the very good agreement of the overall strength of the forward peak (Fig. 8). The few red points in Fig. 7(b), which are systematically higher between 1.5 and $2.3 \mathrm{GeV}$, indicate the different slope observed in this analysis. A less rapid increase of the differential cross section in this kinematic region, $0.3<\cos \theta_{\pi^{0}}$, which was implied by the recent CLAS data and suggested in [4], can thus not be confirmed in this analysis.

Below $E_{\gamma}<1.3 \mathrm{GeV}$, the overall discrepancies between CBELSA/TAPS and CLAS are smaller than about $20 \%$, and thus, both data sets are consistent within systematic errors [Fig. [7(a)]. At higher energies, the forward direction agrees fairly well between the two data sets and appears to be consistent within systematic errors. Only a somewhat higher band of forward points indicates again the steeper slope of the forward peak observed in this analysis. However, agreement with CLAS for $0.6<\cos \theta_{\pi^{0}}$ is fair to good (Fig. 8). Note that additional systematic normalization errors of the CLAS and CB-ELSA data (discussed in 3, 4], but not included in the published data) are not considered in this comparison. Larger systematic differences up to $50 \%$ at and above $E_{\gamma} \approx 1.5 \mathrm{GeV}$ remain only in the backward direction. This confirms the findings of the previous CB-ELSA analysis, which also observed a higher differential cross section in the backward region relative to the CLAS data [Fig. 7(c)]. Older data from Bonn [17] also support these higher cross sections [Fig. [6)(a)].

This forward-backward disagreement suggests a non-trivial scale discrepancy between CLAS and CBELSA/TAPS, and we conclude that a normalization effect can be ruled out. Most of the recent data sets are

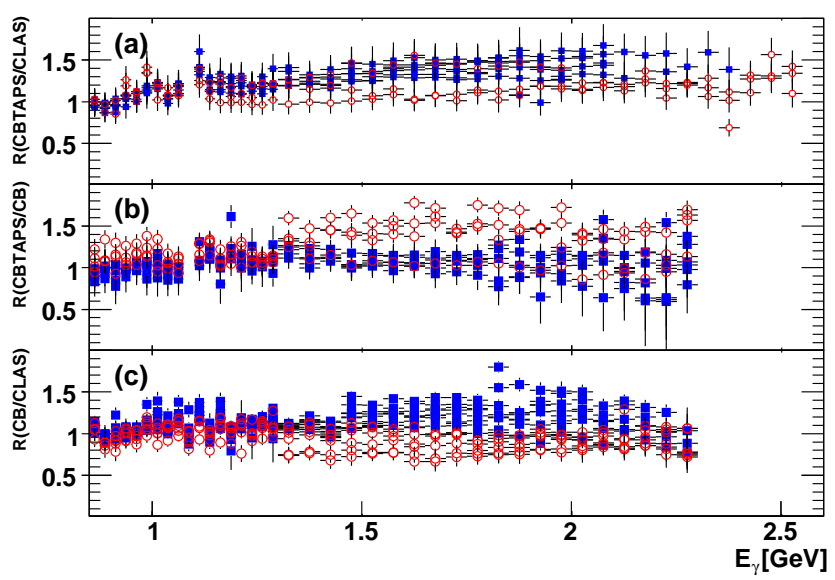

FIG. 7: (Color online) Ratios of differential cross sections from this analysis to CLAS [4] (A) and to CB-ELSA [2] (B), as well as from CB-ELSA to CLAS (C). The red open circles represent the forward direction $\left(\cos \theta_{\pi^{0}}>0\right)$ and the blue squares the backward direction $\left(\cos \theta_{\pi^{0}}<0\right)$. For $E_{\gamma}<1.3 \mathrm{GeV}$, the same CLAS results have been used for the corresponding 25 $\mathrm{MeV}$-wide bins chosen for this analysis. 


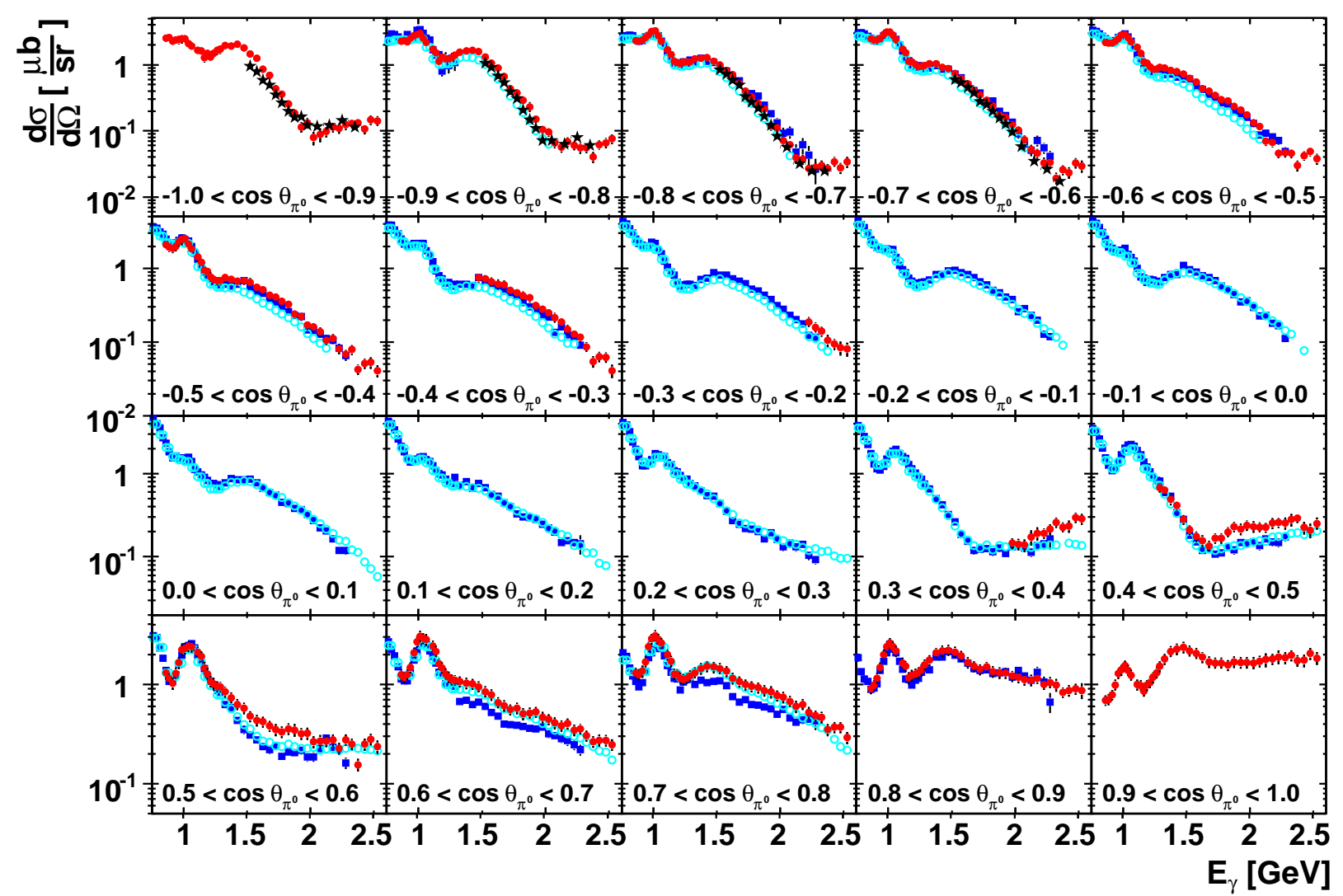

FIG. 8: (Color online) Fixed-angle excitation functions for the reaction $\gamma p \rightarrow p \pi^{0}$; the angle shown is the pion center-ofmass scattering angle. The experimental data are from the new analysis presented here (filled circles •), CB-ELSA [2] (ロ), CLAS [4] (open circles $\bigcirc$ ), and LEPS [16] (filled $\star$ ). Note that the CLAS cross sections were extracted for 50-MeV-wide energy bins; for $E_{\gamma}<1.3 \mathrm{GeV}$, the same CLAS results are shown in the corresponding 25-MeV-wide bins chosen for this analysis.

not statistically limited, and a reason for the observed differences is not known. Since all the discussed discrepancies show the overall level of uncertainty for the reaction $\gamma p \rightarrow p \pi^{0}$ and this has a significant impact on the extraction of resonance couplings, this issue needs to be further addressed and resolved by future experiments.

\section{PARTIAL-WAVE ANALYSIS}

A partial-wave analysis (PWA) has been performed within the Bonn-Gatchina PWA framework and includes the data presented here. For the discussion of contributing resonances, we use the conventional naming scheme of the PDG 27]:

$$
N \text { (mass of the resonance) } L_{2 I, 2 J} \text {, }
$$

where $I$ and $J$ denote isospin and total spin of the resonance, respectively, and $L$ the orbital angular momentum in the decay into a nucleon and a pion. The scattering amplitudes in the PWA for the production and decay of baryon resonances are constructed in the framework of the spin-momentum operator expansion method suggested in 31-33. The approach is relativistically invariant and allows one to perform combined analyses of different reactions imposing analyticity and unitarity directly. The analysis method is further documented in [34, 35]. The experimental database used for the PWA is described in detail in [29] and includes the number of data points for each reaction as well as the weight for each data set used in the fits. It takes into account almost all important sets of photo- and pion-induced reactions, including three-body final states. The latter were included in event-based likelihood fits for the PWA solution discussed in 29]. A full description of the experimental database goes beyond the scope of this paper, and thus we limit the discussion to $\pi^{0}$ photoproduction data.

For the $\gamma p \rightarrow p \pi^{0}$ differential cross sections, data for the invariant mass region above $1600 \mathrm{MeV} / c^{2}$ are available from GRAAL [15], CB-ELSA [2, 3], and CLAS [4]. However, the GRAAL data are available only up to $1950 \mathrm{MeV} / c^{2}$ and the CLAS data cover only a rather restricted angular range at large masses. The new CBELSA/TAPS data cover the forward and backward angular region up to $2370 \mathrm{MeV} / c^{2}$ and therefore provide important information about states in the third and 
fourth resonance regions. In particular, the new data are rather sensitive to contributions from high-spin resonances located in the mass region $2050-2150 \mathrm{MeV} / \mathrm{c}^{2}$. The behavior of the differential cross sections at very extreme angles should be a good indicator for the presence of such states. The sensitivity of the PWA solution to a particular resonance is studied by omitting or including it in the fits and evaluating the fit quality in terms of the resulting $\chi^{2}$ values. The observation of a clear minimum in a mass scan gives further confidence about a contributing resonance. In such a scan, the mass of a resonance is chosen in several steps in the vicinity of the optimum value and fixed in the fit. The mass is then plotted versus the change of the $\chi^{2}$ value from the best fit. Figure 9 shows a typical scan from this analysis.

An overall scaling factor of $14 \%$ is needed in the PWA to achieve quantitative consistency of the data with the previous measurements of the CB-ELSA collaboration [3]. It is worth noting that a similar factor was needed to provide a compatibility between the CB-ELSA and CLAS data [4]. In that case, however, the CLAS data needed to be scaled up by $5 \%$. Fitting only such a scale factor, we obtained a rather good description of the data (reduced $\chi^{2}=1.46$ ) for both solutions reported in [29]. Including the new data in the database and slightly readjusting the parameters, we obtained an improved value of $\chi^{2}=1.26$ without notable changes in the description of other photoproduction data.

The highest spin state which was observed in photoproduction reactions is the $N(2190) G_{17}$ resonance. This is a four-star resonance according to the PDG classification, and the behavior of the $\pi N$ elastic amplitude in this partial wave clearly shows the presence of a pole in the mass region $2100-2200 \mathrm{MeV} / c^{2}$. The recent CLAS data on the reaction $\gamma p \rightarrow K \Lambda$ [36] demand a contribution from this state for a good description of the recoil asymmetry data. Thus, we expect to observe this state in the pion photoproduction reactions. Indeed, we found that the coupling of this state into the $\pi N$ channel (which is fixed by the elastic data) is much stronger than that into the $K \Lambda$ channel, and in the combined analysis of our database, we found a small but not negligible contribution from this state to the pion photoproduction cross section. The pole position of the $G_{17}$ state was found to be $2170-i 155 \mathrm{MeV}$, and the mass scan is shown in Fig. 9 with a solid line. A minimum, fully compatible with that observed in the CLAS $\gamma p \rightarrow K \Lambda$ data is clearly seen.

One of the new states which was observed in the earlier analysis of the pion and $\eta$ photoproduction data [37] was a $D_{13}$ state with a mass of $2166_{-50}^{+25} \mathrm{MeV} / c^{2}$ and a width of $300 \pm 65 \mathrm{MeV} / c^{2}$. In the combined analysis of the present database, this state was optimized with a mass of $2130 \pm 20 \mathrm{MeV} / c^{2}$ and a width of $310 \pm 30 \mathrm{MeV} / c^{2}$. The mass scan of this state for the presented pion photoproduction data is shown in Fig. 9 as a dashed line. It is seen that the minimum corresponds well to the mass of the state found in the combined analysis, but the data are more tolerant to a lower resonance mass.

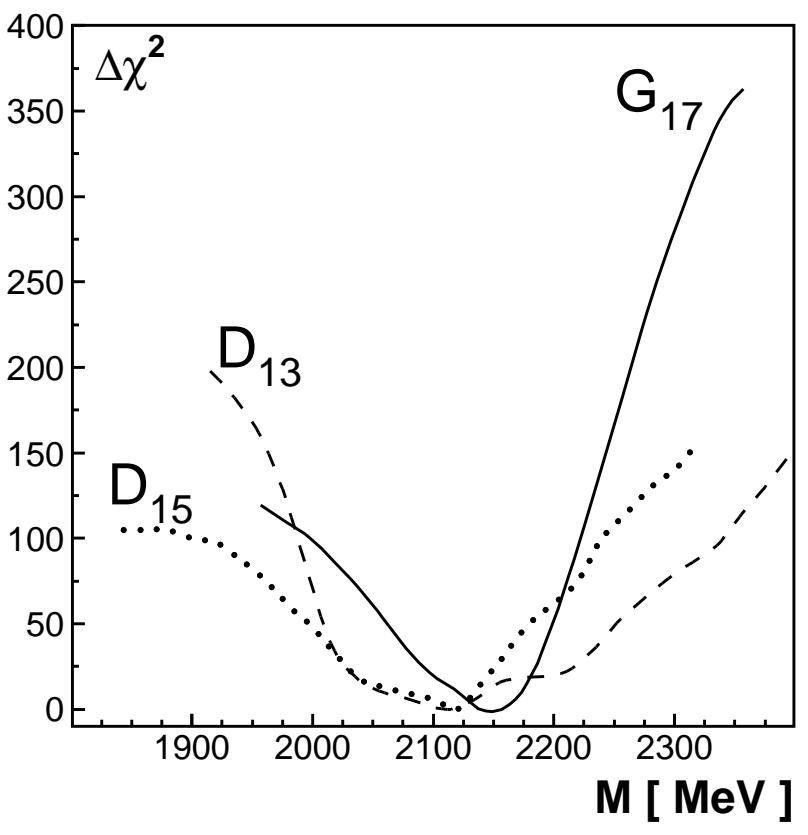

FIG. 9: Mass scans of the $G_{17}$ state (solid line), the second $D_{15}$ state (dotted line), and the fourth $D_{13}$ state (dashed line), which show the deviations in fit quality from the optimum fit $\left(\Delta \chi^{2}\right)$ for a series of mass values.

For the first observation of the $N(2070) D_{15}$ state in the analysis of the $\gamma p \rightarrow p \eta$ differential cross section [37], it was shown that this state can also contribute to the pion photoproduction cross section. In the present analysis, the $\pi N$ coupling of this state was found to be similar to the $\eta N$ coupling. The mass scan, shown in Fig. 9 with a dotted line, produced the minimum slightly above the mass value defined by the combined fit. However, the data can accept well the mass of the state between 2060 and $2120 \mathrm{MeV} / c^{2}$.

Higher-mass $F$-wave states can also contribute to photoproduction and were included as multichannel relativistic Breit-Wigner amplitudes in the PWA [29]. We have performed mass scans of the $N(2000) F_{15}, N(1990) F_{17}$, and $N(1890) S_{11}$ states, which were observed in the analysis of the $\gamma p \rightarrow K \Lambda$ CLAS data [36]. Candidates for these $F$-wave states are listed as two-star resonances by the PDG; a third $S_{11}$ resonance has been assigned a onestar status [27]. Evidence for a higher-mass $F_{15}$-state around $2000 \mathrm{MeV} / \mathrm{c}^{2}$ was also recently reported by the CLAS collaboration in $\omega$ photoproduction off the proton [38]. However, no minima were observed in our mass scans for the description of the present data. Further single- and double-polarization observables are needed to constrain the scattering amplitude and to extract weakly contributing resonances.

In a final comment, we note that our description of the data and the current SAID solution (SP09) [30] underestimate the most-forward data at all three photon energies presented in Fig. 6. The overall systematic error can certainly account for some of the observed discrepancy, but 
some physics may be missing. This needs to be further addressed in future analyses.

\section{SUMMARY}

In summary, we have presented data on the photoproduced $\pi^{0}$ cross section in the reaction $\gamma p \rightarrow p \pi^{0}$. The continuous beam from the ELSA accelerator and the fiber detector of the tagging system provided tagged photons in the energy range from 850 to $2500 \mathrm{MeV}$. The results are in good overall agreement with previous measurements of this reaction but are underestimated by the models at very forward angles for large photon energies. The Bonn-Gatchina PWA found the $N(2190) G_{17}$,
$N(2080) D_{13}$, and $N(2070) D_{15}$ high-mass resonances in its description of the data.

\section{Acknowledgments}

We thank the technical staff at ELSA and at all the participating institutions for their invaluable contributions to the success of the experiment. We acknowledge financial support from the National Science Foundation (NSF), Deutsche Forschungsgemeinschaft (DFG) within the SFB/TR16 and from Schweizerischer Nationalfonds. The collaboration with St. Petersburg received funds from DFG and the Russian Foundation for Basic Research.
[1] E. Klempt and J. M. Richard, Rev. Mod. Phys. 82, 1095 (2010).

[2] O. Bartholomy et al. [CB-ELSA Collaboration], Phys. Rev. Lett. 94, 012003 (2005).

[3] H. van Pee et al. [CB-ELSA Collaboration], Eur. Phys. J. A 31, 61 (2007).

[4] M. Dugger et al., Phys. Rev. C 76, 025211 (2007).

[5] N. Sparks et al. [CBELSA/TAPS Collaboration], Phys. Rev. C 81, 065210 (2010).

[6] M. Yoshioka et al., Nucl. Phys. B 168, 222 (1980).

[7] J. C. Bergstrom, R. Igarashi and J. M. Vogt, Phys. Rev. C 55, 2016 (1997).

[8] R. Beck et al., Phys. Rev. Lett. 65, 1841 (1990).

[9] R. Beck et al., Phys. Rev. Lett. 78, 606 (1997).

[10] M. Fuchs et al., Phys. Lett. B 368, 20 (1996).

[11] B. Krusche et al., Eur. Phys. J. A 6, 309 (1999).

[12] A. Schmidt et al., Phys. Rev. Lett. 87, 232501 (2001).

[13] G. Blanpied et al., Phys. Rev. C 64, 025203 (2001).

[14] J. Ahrens et al. [GDH and A2 Collaborations], Phys. Rev. Lett. 88, 232002 (2002).

[15] O. Bartalini et al. [GRAAL Collaboration], Eur. Phys. J. A 26, 399 (2005).

[16] M. Sumihama et al., Phys. Lett. B 657, 32 (2007).

[17] K. H. Althoff et al., Z. Phys. C 1, 257 (1979).

[18] W. Brefeld, D. Husmann, W. Jansen, B. Lohr, K. Reichmann and H. Schilling, Nucl. Phys. B 100, 93 (1975).

[19] E. Aker et al., Nucl. Instrum. Meth. A 321 (1992) 69.

[20] A. R. Gabler et al., Nucl. Instrum. Meth. A 346, 168 (1994).

[21] R. Novotny [TAPS Collaboration], IEEE Trans. Nucl. Sci. 38, 379 (1991).
[22] W. Hillert, Eur. Phys. J. A 28S1, 139 (2006).

[23] G. Suft et al., Nucl. Instrum. Meth. A 538, 416 (2005).

[24] R. Castelijns et al. [CBELSA/TAPS Collaboration], Eur. Phys. J. A 35, 39 (2008).

[25] M. Nanova et al. [CBELSA/TAPS Collaboration], Eur. Phys. J. A 35, 333 (2008).

[26] V. Crede et al. [CBELSA/TAPS Collaboration], Phys. Rev. C 80, 055202 (2009).

[27] K. Nakamura et al. [Particle Data Group], J. Phys. G 37, 075021 (2010).

[28] C. Amsler et al. [Crystal Barrel Collaboration], Z. Phys. C 58, 175 (1993).

[29] A. V. Anisovich, E. Klempt, V. A. Nikonov, A. V. Sarantsev and U. Thoma, Eur. Phys. J. A 47, 27 (2011).

[30] M. Dugger et al. [CLAS Collaboration], Phys. Rev. C79, 065206 (2009).

[31] A. V. Anisovich et al., J. Phys. G 28, 15-32 (2002).

[32] A. Anisovich, E. Klempt, A. Sarantsev and U. Thoma, Eur. Phys. J. A 24, 111 (2005).

[33] A. V. Anisovich, A. V. Sarantsev, Eur. Phys. J. A 30, 427-441 (2006).

[34] E. Klempt, A. V. Anisovich, V. A. Nikonov, A. V. Sarantsev and U. Thoma, Eur. Phys. J. A 29 (2006) 307.

[35] A. V. Anisovich, V. V. Anisovich, E. Klempt, V. A. Nikonov and A. V. Sarantsev, Eur. Phys. J. A 34 (2007) 129.

[36] M. E. McCracken et al., Phys. Rev. C 81, 025201 (2010).

[37] A. V. Anisovich et al., Eur. Phys. J. A 25, 427 (2005).

[38] M. Williams et al. [ CLAS Collaboration ], Phys. Rev. C 80, 065209 (2009). 